\title{
Iron-responsive element-binding protein 2 plays an essential role in regulating prostate cancer cell growth
}

\author{
Zhiyong Deng ${ }^{1}$, David H. Manz ${ }^{1,2}$, Suzy V. Torti ${ }^{1}$ and Frank M. Torti ${ }^{3}$ \\ ${ }^{1}$ Department of Molecular Biology and Biophysics, UCONN Health, Farmington, Connecticut 06032, USA \\ ${ }^{2}$ School of Dental Medicine, UCONN Health, Farmington, Connecticut 06032, USA \\ ${ }^{3}$ Department of Medicine, UCONN Health, Farmington, Connecticut 06032, USA \\ Correspondence to: Suzy V. Torti, email: storti@uchc.edu \\ Keywords: prostate cancer, iron, iron-responsive element-binding protein, cell cycle, apoptosis \\ Received: April 19, $2017 \quad$ Accepted: June 03, $2017 \quad$ Published: July 17, 2017 \\ Copyright: Deng et al. This is an open-access article distributedunder the terms of the Creative Commons Attribution License 3.0 \\ (CC BY 3.0), which permits unrestricted use, distribution, and reproduction in any medium, provided the original author and source \\ are credited.
}

\section{ABSTRACT}

Iron-responsive element-binding proteins (IRPs) are master regulators of cellular iron homeostasis. Our previous work demonstrated that iron homeostasis is altered in prostate cancer and contributes to prostate cancer progression. Here we report that prostate cancer cells overexpress IRP2 and that overexpression of IRP2 drives the altered iron phenotype of prostate cancer cells. IRP2 knockdown in prostate cancer cell lines reduces intracellular iron and causes cell cycle inhibition and apoptosis. Cell cycle analysis demonstrates that IRP2-depleted prostate cancer cells accumulate in G0/G1 due to induction of p15, p21, and p27. Activation of these pathways is sufficient to significantly reduce the growth of PC3 prostate tumors in vivo. In contrast, IRP1 knockdown does not affect iron homeostasis and only modestly affects cell growth, likely through an iron-independent mechanism. These results demonstrate that upregulation of IRP2 in prostate cancer cells co-opts normal iron regulatory mechanisms to facilitate iron retention and drive enhanced tumor growth.

\section{INTRODUCTION}

Iron is essential for normal cellular metabolism, growth, and replication. Iron and iron-containing functional groups, such as heme and iron-sulfur clusters, are critical to the function of proteins involved in numerous cellular processes including mitochondrial respiration, DNA synthesis, and cell cycle regulation [1]. Excess iron, however, is physiologically detrimental, contributing to generation of reactive oxygen species and oxidative stress [2]. As a result, cellular iron homeostasis is tightly controlled $[3,4]$.

Iron-responsive element-binding proteins (IRPs) are master regulators of cellular iron homeostasis. The two IRPs, IRP1 and IRP2, share $61 \%$ sequence identity [5]. Both IRPs post-transcriptionally regulate iron levels by binding to iron responsive elements (IREs), stem loop structures in the $5^{\prime}$ or $3^{\prime}$ untranslated regions (UTR) of selected mRNAs [6, 7]. IRP/IRE binding in the 5' UTR suppresses mRNA translation while IRP/IRE binding in the 3' UTR stabilizes mRNA transcripts and results in increased translation. 5' IREs are found in mRNAs encoding proteins involved in iron storage and export, such as ferritin and ferroportin (FPN), while 3' IREs are found in mRNAs encoding proteins involved in iron import, such as transferrin receptor (TfR1) and divalent metal transporter 1 (DMT1). Consequently, IRP/IRE binding is normally stimulated in low iron conditions and orchestrates an increase in cellular iron [1].

Prostate cancer is the second leading cause of cancer deaths among men in the United States [8]. Prostate tumors exhibit increased levels of intracellular iron, as do other cancers such as breast [9] and ovarian cancer [10]. Iron import proteins, including TfR1 [11], are overexpressed in prostate cancer cells, while iron export proteins, including FPN, are downregulated [12]. Increased iron retention driven by increased TfR1 and reduced FPN is consistent with increased IRP activity. We therefore hypothesized that IRP overexpression, which occurs in breast cancer [13], drives the altered iron phenotype observed in prostate cancer. 
In this manuscript we demonstrate that overexpression of IRP2, but not IRP1, is a key mechanism by which prostate cancer cells accumulate intracellular iron to drive tumor growth. In vitro, IRP2 knockdown in prostate cancer cell lines reduces intracellular iron, inhibiting cell cycle progression by inducing cell cycle inhibitors, as well as triggering apoptosis. IRP2 knockdown also significantly reduces tumor growth in vivo.

\section{RESULTS}

\section{Transferrin receptor 1 is increased and ferritin $H$ is decreased in prostate cancer cells compared to normal prostate epithelia}

Previous work has shown that prostate cancer cells retain higher levels of metabolically active iron, known as the labile iron pool (LIP), than benign prostate epithelia [12]. Two proteins important for modulation of intracellular iron levels are the iron import protein TfR1 and the iron storage protein ferritin. To determine if these proteins were altered in prostate cancer cells, we compared protein levels of TfR1 and ferritin H (FTH), a subunit of ferritin, in normal prostate epithelial cells (PrEC) and several prostate cancer cell lines (LNCaP, VCaP, PC3, and DU145). As illustrated in Figure 1A, TfR1 protein was markedly increased in all prostate cancer cell lines as compared to normal prostate epithelial cells. In contrast, FTH protein levels were decreased to barely detectable levels in prostate cancer cells. Elevated TfR1 and downregulated ferritin are associated with a high iron uptake and low iron storage capability.

\section{IRP2 is upregulated in prostate cancer cells}

The increase in TfR1 and decrease in ferritin observed in prostate cancer cells appear to contradict what is known about iron regulatory mechanisms. In particular, in the classic model of iron regulation, high levels of intracellular iron are expected to inactivate iron regulatory proteins (IRP1 and IRP2), which in turn would decrease TfR1 and increase ferritin through post-transcriptional mechanisms [14]. However in prostate cancer cells, we observed a decrease in ferritin and increase in TfR 1 (Figure 1), despite the increased labile iron pool in these cells [12]. To explain this paradox, we hypothesized that prostate cancer cells might constitutively over-express either IRP1 or IRP2, and that overexpression of these proteins might over-ride the classic iron-mediated iron regulatory pathway to permit high levels of TfR 1 and low levels of ferritin in these iron replete cells.

To test this hypothesis, we measured levels of IRP1 and IRP2 in these cells. As shown in Figure 1A, IRP2 was consistently overexpressed in prostate cancer cell lines, with $4 / 4$ cell lines exhibiting elevated levels of
IRP2 when compared to normal prostate epithelial cells. In contrast, IRP1 protein levels were elevated in only two of four prostate cancer cell lines. Mirroring these changes at the protein level, mRNA levels of IRP2 were consistently upregulated in prostate cancer cells (Figure 1B). Levels of IRP1 were generally not increased, with only LNCaP cells exhibiting a modest increase in IRP1 (Figure 1C). Taken together, these results suggested IRP2 rather than IRP1 as a critical contributor to the altered iron phenotype of prostate cancer cells.

\section{IRP2 is critical for altered iron homeostasis in prostate cancer cells}

To determine if IRP2 overexpression drives the iron phenotype of prostate cancer cells, we depleted IRP2 in LNCaP cells using two independent lentiviral shRNAs (Figure 2A). Following IRP2 knockdown, LNCaP cells expressed dramatically reduced TfR 1 and increased FTH (Figure 2A). Importantly, we observed no effect of IRP2 shRNAs on IRP1 (Figure 2A). In PC3 cells, IRP2 knockdown produced similar effects (Figure 2B). To confirm a functional effect of TfR1 downregulation in shIRP2 cells, we measured uptake of fluorophore-labeled transferrin by flow cytometry in LNCaP shControl and shIRP2 cells. As expected, LNCaP sh-IRP2 cells exhibited reduced transferrin uptake compared to shControl cells (Figure 2C), demonstrating a diminished ability to import transferrin-bound iron. To further confirm that IRP2 knockdown reduced iron retention in prostate cancer cells, we measured the labile iron pool (LIP) following IRP2 knockdown in LNCaP and PC3 cells. Consistent with our other findings, IRP2 knockdown significantly depleted LIP levels in both cell lines (Figure 2D and 2E), confirming that overexpression of IRP2 drives iron retention in prostate cancer cells.

\section{Reduction in IRP2 inhibits prostate cancer cell proliferation in vitro}

We next sought to determine the role of IRP2 overexpression in prostate cancer cell proliferation. We compared proliferation rates in control and IRP2depleted cells by direct cell counts. As shown in Figure $3 \mathrm{~A}$ and $3 \mathrm{~B}$, reduction of IRP2 suppressed proliferation in $\mathrm{LNCaP}$ and $\mathrm{PC} 3$ prostate cancer cells. These results were confirmed using metabolic (WST-1) (Figure 3C and 3D) and clonogenic assays (Figure $3 \mathrm{E}$ and $3 \mathrm{~F}$ ). Thus, IRP2 overexpression facilitates prostate cancer cell proliferation.

\section{IRP1 knockdown has minimal effects on prostate cancer cell proliferation}

These data, supported by previous in vivo and cellular studies [15, 16], suggested that IRP2, rather than IRP1 plays a predominant role in regulation of iron 
metabolism. To directly evaluate the contribution of IRP1 in regulating prostate cancer iron metabolism and cell growth, we utilized two distinct shRNAs to knockdown IRP1 in LNCaP cells. As shown in Figure 4A, there were no appreciable changes in TfR1, FTH, or IRP2 protein following IRP1 knockdown. We then assessed the effects of IRP1 knockdown on cell growth as compared to shControl and sh-IRP2 cells (Figure 4B). IRP1 knockdown led to a modest reduction in cell proliferation rate compared to IRP2 knockdown. These results support a greater dependence of prostate cancer cell growth on IRP2 than IRP1. Further, it is unlikely that the effect of IRP1 knockdown on cell growth was a result of altered iron metabolism, since manipulation of IRP1 did not alter expression of other iron proteins (Figure 4A).

\section{IRP2 knockdown regulates cell cycle in prostate cancer cells}

Having confirmed that IRP2 knockdown has a pronounced effect on prostate cancer cell proliferation, we sought to identify the mechanism by which cell proliferation is inhibited. We first tested whether iron depletion following IRP2 knockdown resulted in cell cycle inhibition. We labeled control and IRP2 knockdown LNCaP and PC3 cells with propidium iodide and examined cell cycle phase distribution using flow cytometry (Figure $5 \mathrm{~A}$ and $5 \mathrm{~B}$ ). In both cell lines we observed a significant accumulation of cells in G0/G1 phase following IRP2 knockdown. In LNCaP IRP2 knockdown cells, the increase of cells in G0/G1 phase was accompanied by a significant decrease in cells in $\mathrm{S}$ phase (Figure 5A). Similarly, a decrease in the number of cells in $\mathrm{S}$ phase was observed following IRP2 knockdown in PC3 cells, although the decrease was not statistically significant. PC3 cells also demonstrated a small decrease in the number of cells in G2/M following IRP2 knockdown (Figure 5B).

Despite some differences, IRP2 knockdown in both LNCaP and PC3 cells resulted in accumulation of cells in $\mathrm{G} 0 / \mathrm{G} 1$. To determine the mechanism responsible for G0/G1 arrest in IRP2 knockdown cells, we examined transcript levels of the G0/G1 cell cycle checkpoint proteins p15 (CDKN2B), p21 (CDKN1A), and p27 (CDKN1B) by real-time qPCR. As shown in Figure $5 \mathrm{C}$, these cell cycle regulating genes were upregulated following IRP2 knockdown in LNCaP cells, consistent

A

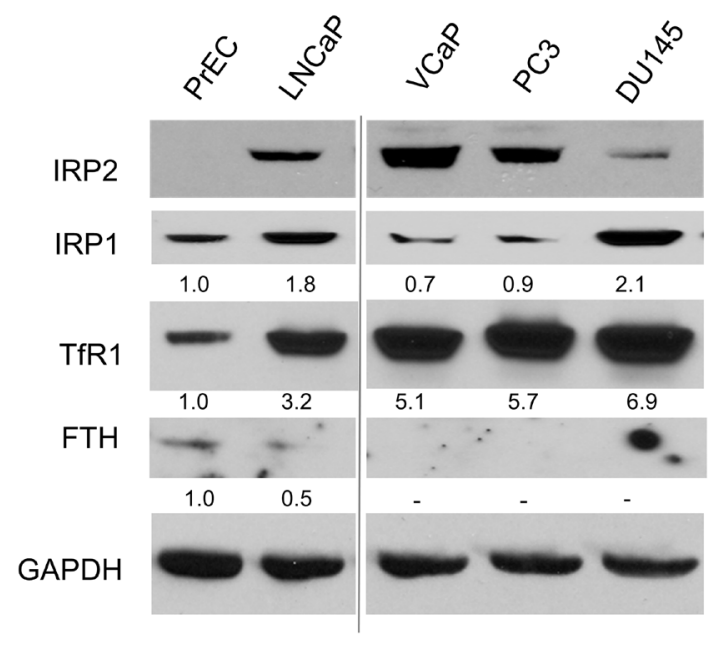

B

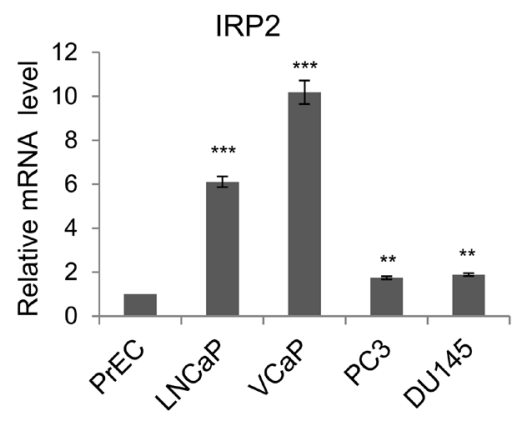

C

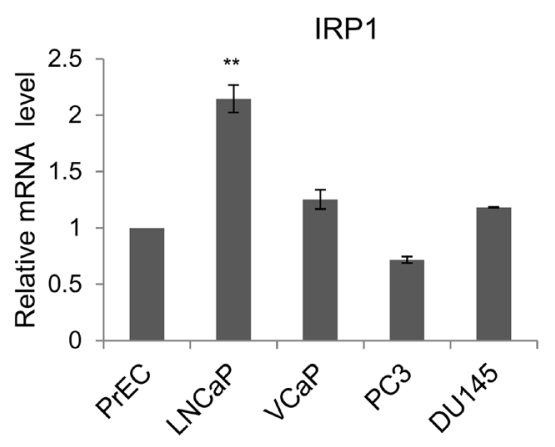

Figure 1: IRP2 is overexpressed in prostate cancer cells. (A) Western blot of transferrin receptor 1 (TfR1), ferritin H (FTH) (monoclonal antibody detection), iron regulatory protein 1 (IRP1), iron regulatory protein 2 (IRP2), and GAPDH (loading control) in normal prostate epithelia (PrEC) and prostate cancer cells (LNCaP, VCaP, PC3, DU145). (B, C) Relative mRNA levels of (B) IRP2 and (C) IRP1 detected by real-time qPCR. Data are representative of 3 independent experiments $\left(* \mathrm{p}<.05, * * \mathrm{p}<.01,{ }^{* * *} \mathrm{p}<.001\right)$. 
with cell cycle inhibition. Upregulation of p15, p21 and p27 protein following IRP2 knockdown in these cells was confirmed by western blot (Figure 5D).

While many genes regulated by the IRP-IRE system are involved in iron metabolism, IRPs have also been reported to regulate genes involved in other cellular processes [17]. To confirm that the effects of IRP2 knockdown on cell growth and p15, p21, and p27 were caused by iron depletion, we tested whether these changes could be mimicked by depleting iron with the iron chelator desferoxamine (DFO). LNCaP cells treated with DFO showed a dose-dependent reduction in cell proliferation (Supplementary Figure 1A) and recapitulated the induction of p15, p21, and p27 (Supplementary Figure 1B). Taken together, these data indicate that prostate cancer cells overexpress IRP2 to maintain adequate iron levels to support rapid cell cycling. When IRP2 is reduced, prostate cancer cell proliferation is impaired by cell cycle inhibitors p15, p21, and p27.

A

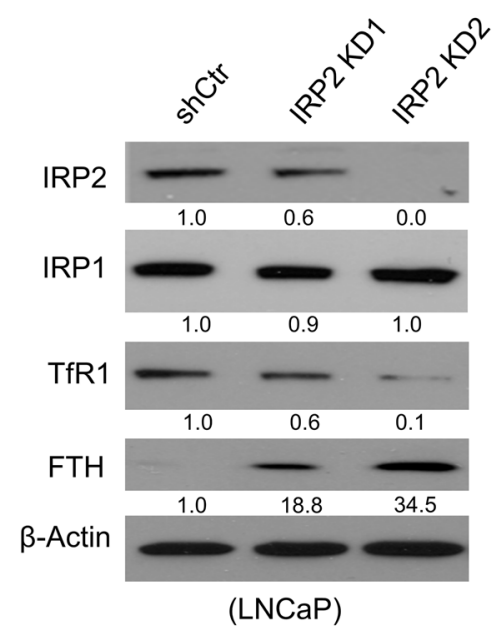

C

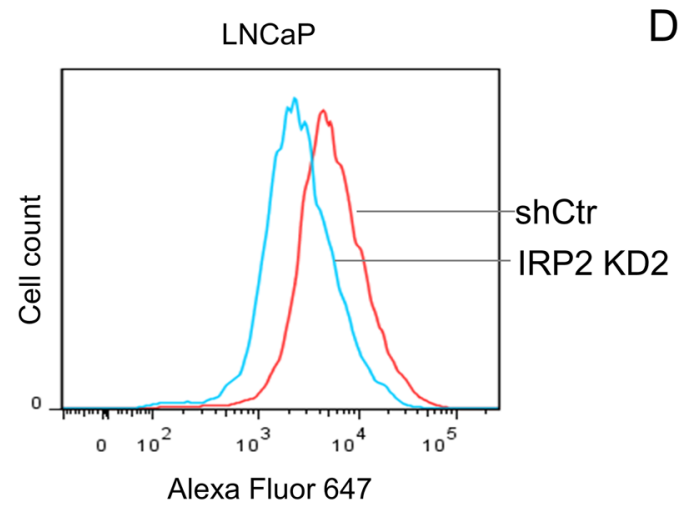

\section{Apoptosis is induced by IRP2 depletion in prostate cancer cells}

Cell cycle checkpoint proteins are involved in both cell cycle regulation and initiation of apoptotic death. As iron deficiency has been shown to induce apoptosis [18], we sought to determine if IRP2 depletion also induced apoptosis of prostate cancer cells. Visual inspection of cell cultures revealed an increase in detached cells, a sign of cell death, following IRP2 knockdown in prostate cancer cells. To test if IRP2 knockdown triggered apoptosis, we measured the activity of caspase $3 / 7$ in LNCaP and PC3 cells. We found that IRP2 knockdown increased caspase $3 / 7$ activity in both cell lines (Figure 6A and 6B). We next determined the proportion of apoptotic cells by flow cytometry using 7-AAD/Annexin V staining [19]. As demonstrated in Figure $6 \mathrm{C}$ and $6 \mathrm{D}$, Annexin $\mathrm{V}$ singlestained (early apoptotic) and Annexin V/7AAD doublestained (late apoptotic) cells were both significantly
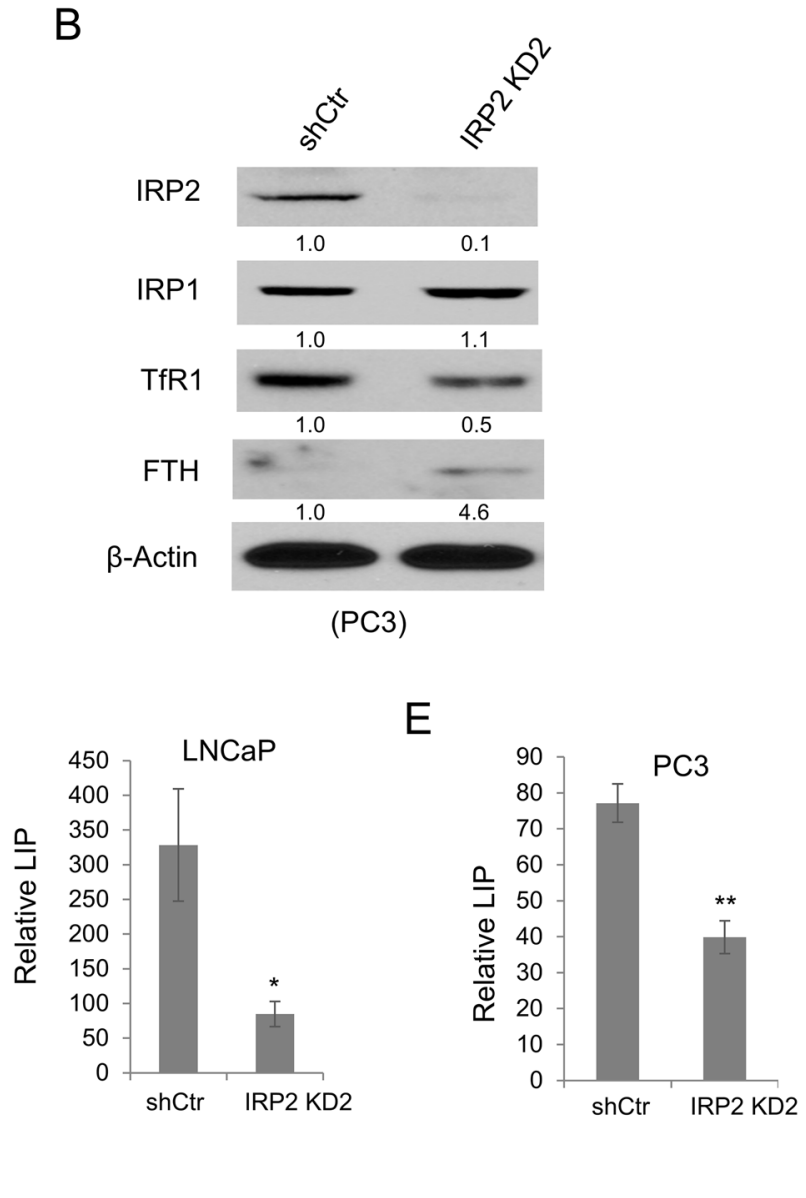

Figure 2: IRP2 knockdown alters prostate cancer iron metabolism and reduces intracellular iron level. (A, B) Western blot of iron regulatory protein 2 (IRP2), iron regulatory protein 1 (IRP1), transferrin receptor 1 (TfR1), ferritin H (FTH) (monoclonal antibody detection), and $\beta$-actin (loading control) in (A) LNCaP and (B) PC3 cells infected with lentiviral IRP2-shRNA(s) (IRP2 KD) and scrambled control shRNA (shCtr). (C) Alexa Fluor647-conjugated transferrin uptake assessed by flow cytometry in shCtr and IRP2 KD LNCaP cells. (D, E) Labile iron pool (LIP) levels in (D) LNCaP and (E) PC3 shCtr and IRP2 KD cells. Data are representative of 3 independent experiments $(* \mathrm{p}<.05, * * \mathrm{p}<.01)$. 
increased following IRP2 knockdown in LNCaP and PC3 cells. These results indicate that IRP2 overexpression is an important determinant of cell fate that allows prostate cancer cells to avoid apoptosis.

\section{IRP2 depletion attenuates the growth of prostate cancer xenografts}

We next utilized a xenograft model to assess the role of IRP2 in prostate cancer growth in vivo. PC3 cells expressing a stable luciferase construct (PC3/Luc) were generated to facilitate tracking of tumor cells in vivo. These cells were then infected with sh-IRP2 or shControl lentiviruses. We confirmed knockdown of IRP2 in PC3/ Luc sh-IRP2 cells prior to starting the experiment (Figure 7A). PC3/Luc shControl and sh-IRP2 cells were inoculated into the flanks of nude mice and tumors allowed to grow for 15 days prior to initiating measurements of tumor size. IRP2 knockdown significantly reduced tumor volume beginning at day 29 as assessed by both caliper measurement (Figure 7A) and bioluminescent imaging (Figure 7B). These findings show that IRP2 depletion can suppress prostate tumor growth in vivo.

\section{DISCUSSION}

Growing evidence demonstrates that iron metabolism is remodeled in numerous cancer types to enable enhanced iron acquisition and retention $[9,12$, 13]. Indeed, a recent bioinformatics analysis revealed that expression of nearly half of 61 genes involved in some aspect of iron metabolism were associated with distant
A

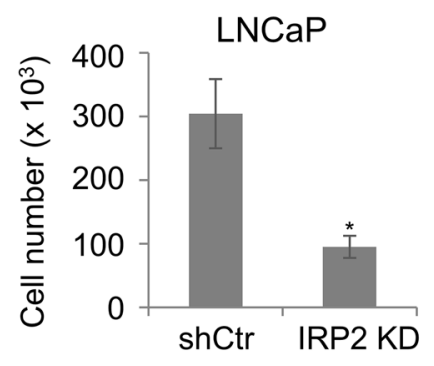

C

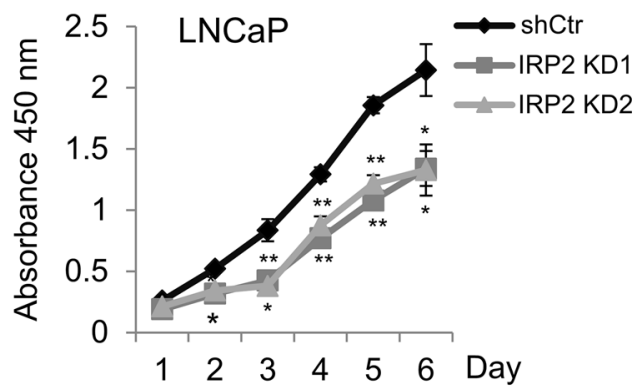

$E$

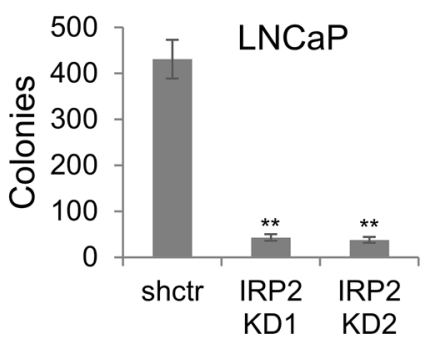

B

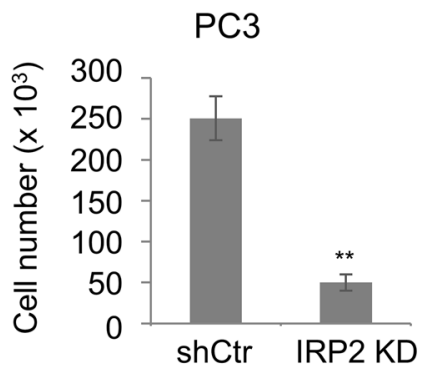

$\mathrm{D}$

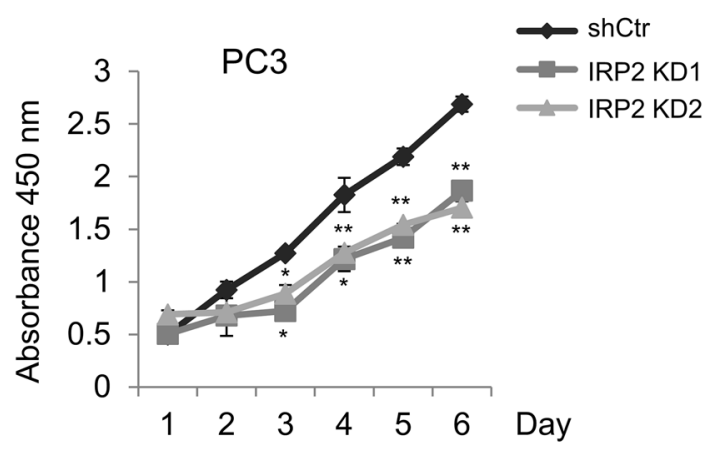

$\mathrm{F}$

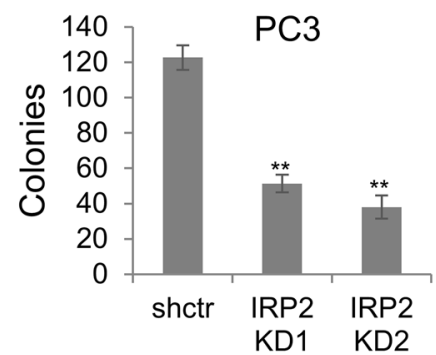

Figure 3: IRP2 knockdown inhibits proliferation of prostate cancer cells. (A, B) Cell count by hemocytometer of (A) LNCaP and (B) PC3 shCtr and IRP2 KD cells after 7 days. (C, D) WST-1 proliferation assay of (C) LNCaP and (D) PC3 shCtr and IRP2 KD cells. (E, F) Clonogenic assays for (E) LNCaP (10 days) and (F) PC3 cells (12 days). Data are representative of 3 independent experiments $(* \mathrm{p}$ $<.05, * * \mathrm{p}<.01)$. 

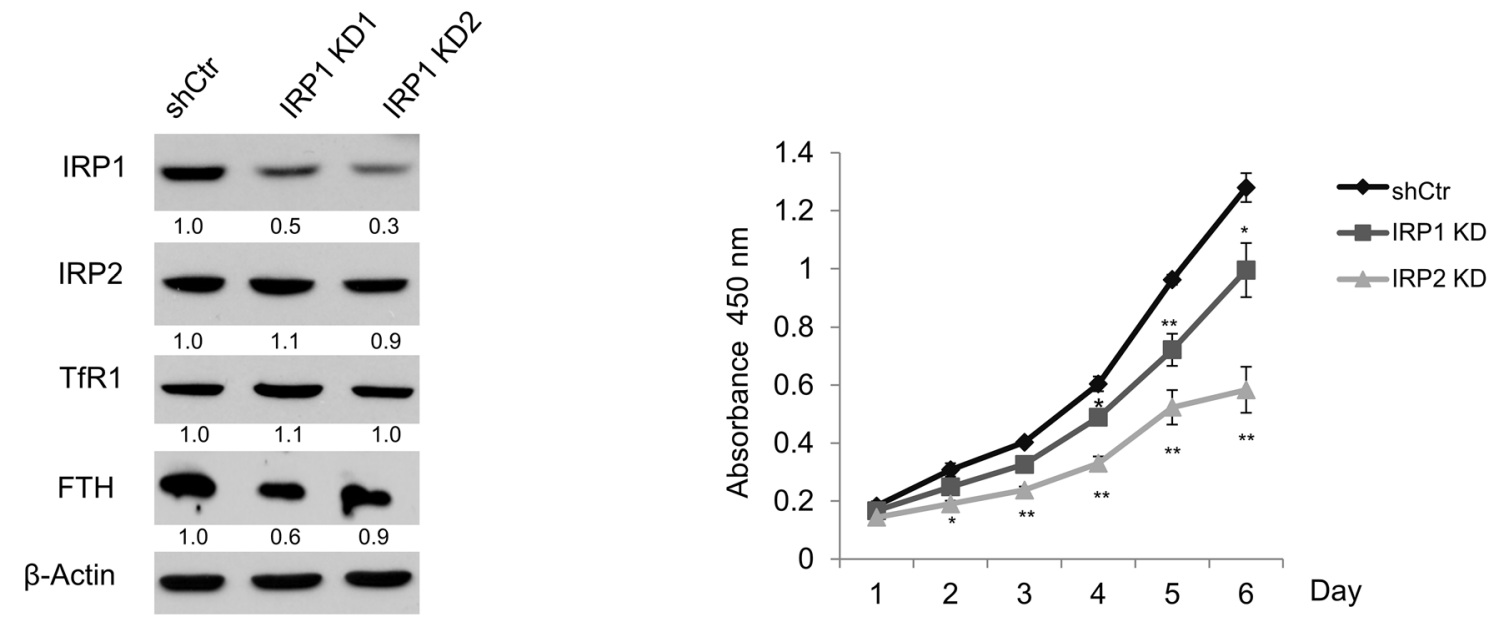

Figure 4: IRP1 silencing does not affect expression of iron proteins and only modestly inhibits proliferation of LNCaP cells. (A) Western blot of iron regulatory protein 1 (IRP1), iron regulatory protein 2 (IRP2), transferrin receptor 1 (TfR1), ferritin $\mathrm{H}$ (FTH), and $\beta$-actin (loading control) in LNCaP cells infected with lentiviral IRP1-shRNAs (IRP1 KD1 and KD2) and scrambled control shRNA (shCtr). A polyclonal FTH antibody with increased sensitivity was used for this experiment [47]. (B) WST-1 proliferation assay of LNCaP shCtr, IRP1 KD, and IRP2 KD cells. Data are representative of 3 independent experiments $\left(* \mathrm{p}<.05,{ }^{* *} \mathrm{p}<.01\right)$.

A

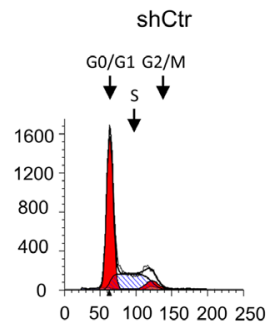

B

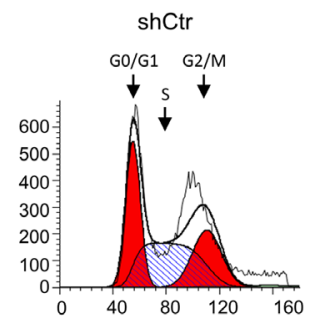

C

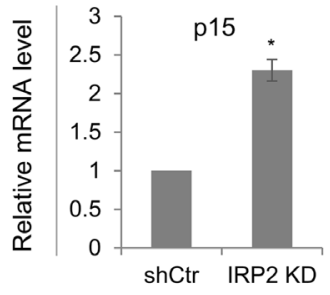

IRP2 KD
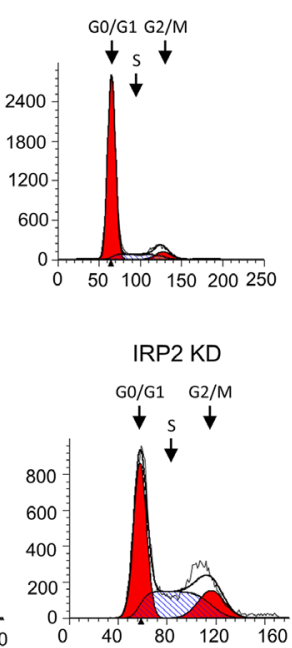
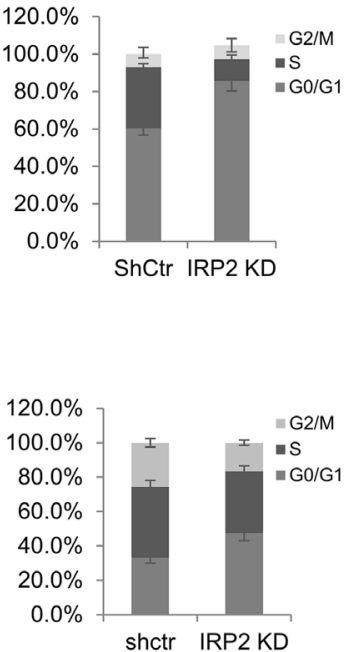
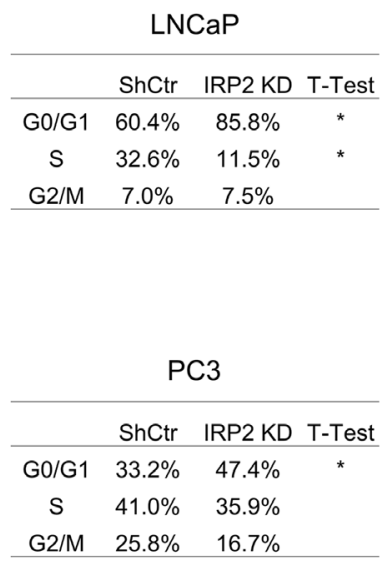

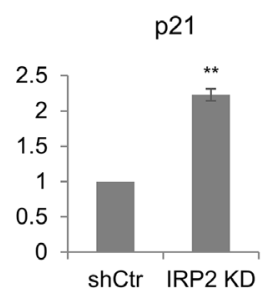

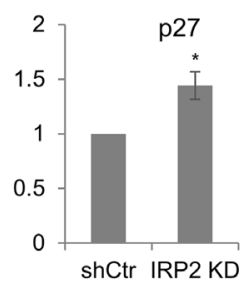

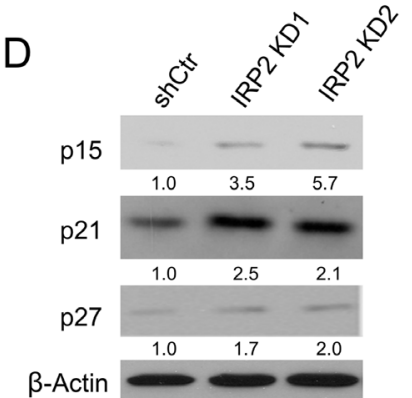

Figure 5: IRP2 knockdown modulates cell cycle regulators and inhibits cell cycle progression. (A, B) DNA content of propidium iodide stained (A) LNCaP and (B) PC3 control (shCtr) and IRP2 knockdown (IRP2 KD) cells assessed by flow cytometry. Cell cycle distribution was analyzed by ModFit LT software. (C) Relative mRNA levels of p15, p21, and p27 in LNCaP shCtr and IRP2 KD cells assessed by real-time qPCR. (D) Western blot of p15, p21 and p27 in LNCaP shCtr and IRP2 KD cells. Data are representative of 3 independent experiments $(* \mathrm{p}<.05, * * \mathrm{p}<.01)$. 
metastasis-free survival in breast cancer [20]. While these findings suggest that iron dysregulation promotes tumor progression, the mechanisms by which alterations in iron metabolism are implemented and their effects on tumor behavior remain incompletely understood.

We therefore explored the role of the only two known mammalian IRPs, IRP1 and IRP2, which are master regulators of cellular iron. IRP1 and IRP 2 share an overall amino acid identity of approximately $61 \%$ [5] and both have high affinity for IREs. Despite these similarities, IRP1 and IRP2 have been reported to execute different cellular functions through regulation of non-overlapping mRNA targets $[17,21]$. Our findings support a functional difference between IRP1 and IRP2 by demonstrating that IRP2 is specifically co-opted by prostate cancer cells to alter normal iron homeostasis and retain increased intracellular iron (Figure 2). IRP1 was unable to alter ferritin and TfR1 when depleted by shRNA knockdown
(Figure 4), suggesting that the modest effect of IRP1 knockdown on cell growth is likely iron-independent. In contrast, IRP2 knockdown inhibited prostate cancer cell growth in an iron-dependent fashion (Figures 2 and 3 ). These findings are consistent with previous reports that IRP2 plays a predominant role over IRP1 in iron homeostasis in vitro [16] and in vivo [15].

Our findings demonstrate that constitutive overexpression of IRP2 is broadly observed in prostate cancer. Overexpression of IRP2 was consistently observed in all prostate cancer cell lines studied (Figure 1). IRP2 protein is tightly regulated by FBXL5mediated proteasomal degradation [22-24] as well as non-proteasomal degradation [25]. While it is possible that prostate cancer cells drive IRP2 overexpression by altering post-transcriptional regulation, our findings demonstrate that IRP2 is transcriptionally upregulated in prostate cancer cells (Figure 1). We speculate that $c-m y c$,
A

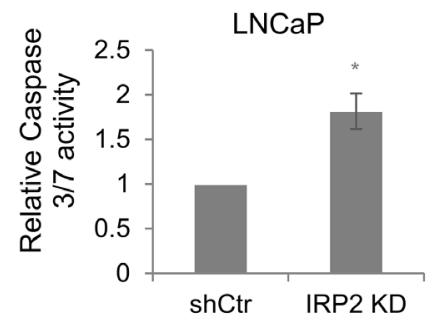

C

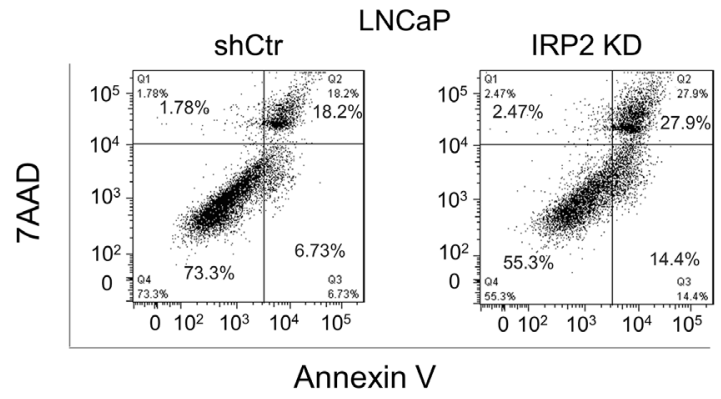

D

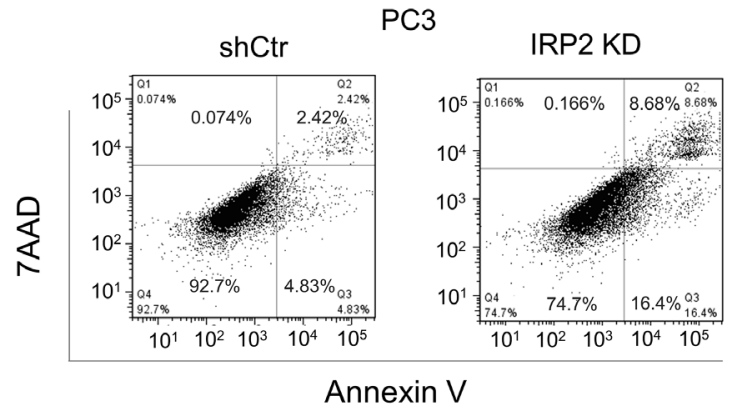

B
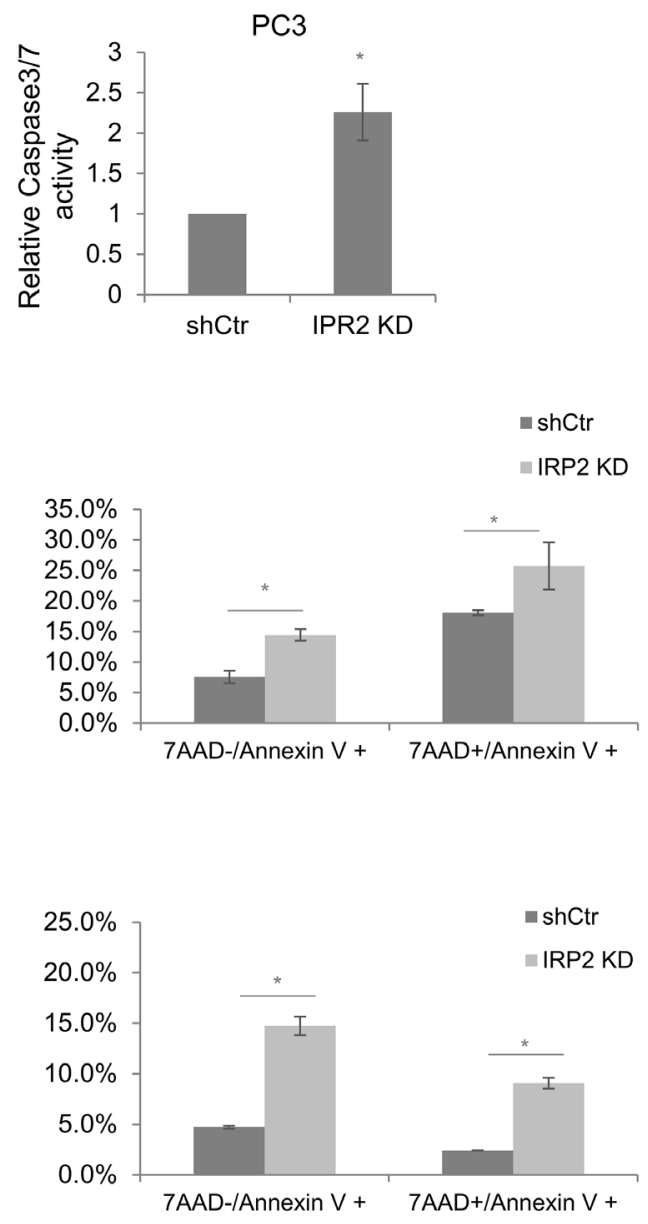

Figure 6: IRP2 knockdown induces apoptosis. (A, B) Caspase-Glo 3/7 assay in (A) LNCaP and (B) PC3 control (shCtr) and IRP2 knockdown (IRP2 KD) cells. (C, D) Annexin V and 7-aminoactinomycin D (7AAD) staining in (C) LNCaP and (D) PC3 cells shCtr and IRP2 KD cells measured by flow cytometry. Bar graphs indicate the quantification of early apoptotic (7AAD-/Annexin $\mathrm{V}+$ ) and late apoptotic cells (7AAD+/Annexin $\mathrm{V}+)(* \mathrm{p}<.05)$. 
an oncogene that is frequently misregulated in prostate cancer $[26,27]$ and is a known transcriptional regulator of IRP2 [28], may be the driver of IRP2 overexpression in some prostate tumors. Future experiments will be required to assess whether this or other mechanisms drive IRP2 overexpression in prostate cancer.

Overexpression of IRP2 may enable prostate cancer cells to bypass normal feedback loops that are designed to limit iron uptake in cells that are iron replete. In noncancer cells, IRPs are inactivated under conditions of high iron, triggering a compensatory decrease in TfR1 and increase in ferritin [14]. Prostate cancer cells that overexpress IRP2 bypass this checkpoint and express high levels of TfR 1 and low levels of ferritin despite high levels of intracellular iron (Figure 1). Overexpression of IRP2 is thus a mechanism enabling the increased iron uptake and retention that characterizes cancer cells. To test the dependence of prostate cancer cells on this pathway, we depleted IRP2 in prostate cancer cells using lentiviral shRNA. IRP2 knockdown dramatically altered the iron regulatory proteins $\mathrm{TfR}$ and ferritin, reducing intracellular iron (LIP) levels (Figure 2). IRP2-induced LIP reduction

\section{A}
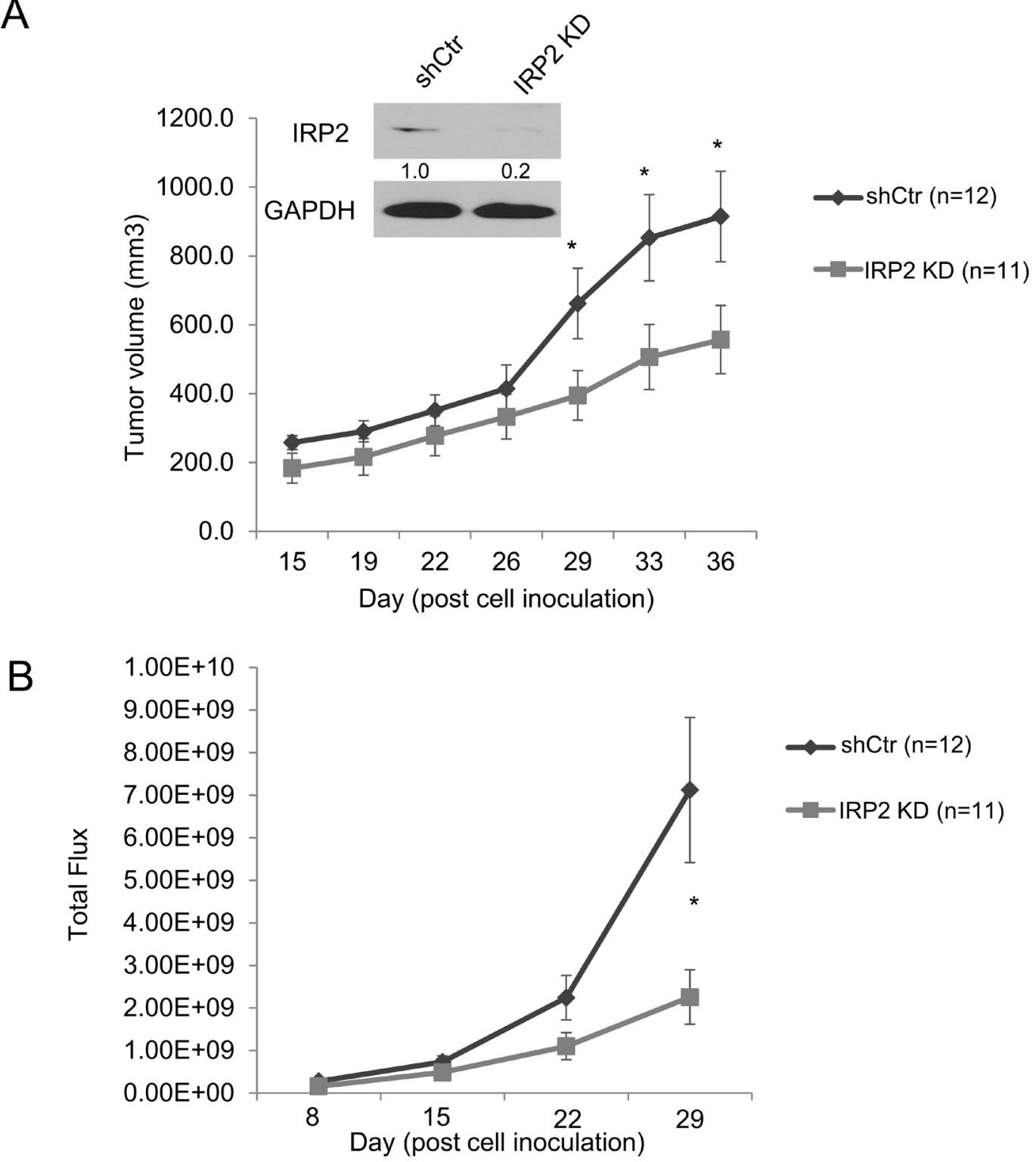

Figure 7: IRP2 deficiency inhibits tumor growth of prostate cancer cells in vivo. Luciferase expressing PC3 (PC3/Luc) cells were infected with lentiviral scrambled control shRNA (shCtr) or IRP2-shRNA (IRP2 KD). 2 x 106 PC3/Luc cells were inoculated into flanks of nude mice by subcutaneous injection. (A) Western blot of iron regulatory protein 2 (IRP2) and GAPDH (loading control) at day 0 . Tumor growth was evaluated by caliper measurement at 15, 19, 22, 26, 29, 33, and 36 days post-inoculation. (B) Tumor growth was evaluated by bioluminescent luciferase imaging at $8,15,22$, and 29 days post-inoculation. Data are means \pm Standard Error with $n=11 / 12$ $(* \mathrm{p}<.05)$. 
was sufficient to inhibit prostate cancer cell proliferation, both in vitro (Figure 3) and in vivo (Figure 7), and induce apoptosis (Figure 6).

Because cell cycle checkpoints link cell cycle progression and apoptotic cell death [29], we investigated the effects of IRP2 knockdown on these proteins. We determined that IRP2 knockdown induced cell cycle checkpoint proteins p15, p21, and p27 (Figure 5C and 5D), consistent with the observed G0/G1 cell cycle arrest (Figure 5A and 5B).

While IRP2 knockdown resulted in G0/G1 arrest in both LNCaP and PC3 cells, the magnitude of cell cycle arrest differed, as did the distribution of cells in $\mathrm{S}$ and G2/M phases. Thus, LNCaP IRP2 KD cells exhibited a robust $\mathrm{G} 0 / \mathrm{G} 1$ arrest and an attendant decrease in the percentage of cells in S phase, whereas PC3 IRP2 KD cells showed a moderate $\mathrm{G} 0 / \mathrm{G} 1$ arrest and a decrease in the percentage of cells in $\mathrm{G} 2 / \mathrm{M}$ phase (Figure 5). It is likely that the observed differences in the effects of IRP2 on the cell cycle in these two cell lines is a result of differences in their genetic backgrounds, since LNCaP and PC3 cells derive from quite different prostate tumors [30-32]. In particular, LNCaP cells express wildtype-p53, whereas PC3 cells lack functional p53 due to a nonsense mutation [33]. p53 is known to induce $\mathrm{G} 0 / \mathrm{G} 1$ arrest and can also play a role in $\mathrm{G} 2 / \mathrm{M}$ arrest [34]. p53 was induced by IRP2 knockdown in LNCaP cells (Supplementary Figure 2), suggesting that p53 status may contribute to the enhanced G0/G1 arrest observed in LNCaP cells when compared to PC3 cells. Other differences between these two cell lines may also play a role [35]. Importantly, these findings demonstrate that iron depletion by IRP2 overexpression is an effective method for inhibiting growth of biologically diverse prostate cancer cell types.

Given the demand of cancer cells for iron, iron depletion may be a promising therapeutic strategy to restrict tumor growth. Iron chelators are currently being evaluated as anti-cancer agents [36], as are irontargeting gallium compounds [37]. Our finding that IRP2 knockdown in prostate cancer cells recapitulates the effects of iron chelators on cell cycle inhibition $[38,39]$ (Figure 5) and induction of apoptosis [40-43] (Figure 6) suggests that targeting IRP2 may represent an alternative approach to restricting tumor growth by targeting iron. The substantial IRP2 overexpression in cancer cells suggests that there may be a favorable therapeutic window for IRP2-targeted treatments. Further, because IRP2-targeted therapy has the potential to alter multiple facets of cellular iron homeostasis, including iron uptake, storage, utilization, and export, inhibiting IRP2 may be more effective than blocking individual components of iron metabolism, such as TfR1 [44]. For these reasons, IRP2 may represent a promising target for cancer therapy.

\section{MATERIALS AND METHODS}

\section{Cell culture}

Cell culture reagents were obtained from Gibco (Life Technologies, Carlsbad, CA) unless otherwise specified. The human prostate cancer cell lines LNCaP, PC3, and DU145 were purchased from American Type Culture Collection (ATCC). Cells were grown in RPMI 1640 medium containing $10 \%$ fetal calf serum and $1 \%$ PenStrep and incubated at $37^{\circ} \mathrm{C}$ in a humidified atmosphere containing $5 \% \mathrm{CO}_{2}$. $\mathrm{VCaP}$ cells were provided by Dr. Donna Peehl (Stanford University) and cultured in Dulbecco's Modified Eagle's Medium (DMEM) containing $10 \%$ fetal calf serum and $1 \%$ PenStrep. Normal prostate epithelial cells (PrEC) were purchased from Lonza and cultured in Prostate Cell Growth Medium (Lonza, CC3166). Cells were studied at a low passage number (less than 30 thaw-freeze cycles after thaw from early-passage frozen stocks). Cell authentication was performed by ATCC based on short-tandem-repeat (STR) profiling and cell morphological features were routinely monitored.

\section{DNA vectors}

Lentiviral shRNA constructs were constructed on a previously described shRNA vector backbone using an established protocol $[45,46]$. Targeted shRNA vectors were generated using the following target sequences: 5'-GATCTTACAGTTGACCATTCT-3' (shRNAIRP2-\#1), 5'-GGAGTGGCTGGAAAGTTTGTT -3' (shRNA-IRP2-\#2), 5'-GTAATAGCATATGCAATTG CT-3'(shRNA-IRP1-\#1), and 5'-GAACGATACACTATCA TTATT-3' (IRP1-shRNA-\#2). The non-targeted, scrambled shRNA control was previously validated [46]. A lentiviral luciferase expression vector was constructed by subcloning the LUC2 gene and zeocin resistance gene from the pGL4 vector (Promega Co., Madison, WI, USA) into the pLKO.1-shCtr vector (Addgene catalog \#10879) (34). First, the shRNA expression cassette was removed and the puromycin resistance gene was supplanted with the LUC2 coding region, an internal ribosome entry site, and the zeomycin resistance gene. These modifications resulted in a vector expressing luciferase and zeocin driven by the original phosphoglycerate kinase (PGK) promoter. shRNA knockdown experiments were performed 5-7 days after infection.

\section{Cell proliferation assays}

Cell proliferation was measured manually by hemocytometer cell counting with trypan blue staining to exclude non-viable cells. 8-16 quadrants were counted. Individuals performing these measurements were not blinded to the identity of the samples. A colorimetric assay using the tetrazolium salt 4-[3-(4-lodophenyl)-2- 
(4-nitrophenyl)-2H-5-tetrazolio]-1,3-benzene disulfonate (WST-1) (Roche) was also used to measure cellular proliferation following the manufacturer's instructions. Clonogenic assays were performed for three days following lentiviral shRNA infection. Cells were seeded in 6-well plates at densities of 800 ( $\mathrm{LNCaP})$ or 400 (PC3) cells per well. Cells were then fixed in $10 \%$ formalin after 10 days (LNCaP) or 12 days (PC3) and stained with $0.25 \%$ crystal violet to detect colony formation.

\section{Cell cycle analysis}

Cells were harvested and washed twice with PBS and the cell pellets were suspended at a concentration of 1 $\mathrm{x} 10^{6} \mathrm{cells} / \mathrm{ml}$. Cells were then gently vortexed to minimize clumping, meanwhile three volumes of cold $\left(-20^{\circ} \mathrm{C}\right)$ absolute ethanol were added to fix the cells at $-20^{\circ} \mathrm{C}$ for at least 1 hour. Fixed cells were further washed twice with PBS and mixed with an appropriate volume of FxCycle ${ }^{\mathrm{TM}}$ PI/RNase Staining Solution (Life Technologies, Carlsbad, CA) to stain nuclear DNA. Stained cells were maintained at room temperature for 15-30 minutes to ensure complete staining and minimize RNA contamination. Cell cycle analysis was performed on a MACSQuant flow cytometer (Miltenyi Biotec, Bergisch Gladbach, Germany). The data were analyzed with ModFit LT 3.0 software (Verity Software House, Topsham, ME, USA).

\section{Apoptosis assay}

The Caspase-Glo 3/7 assay kit (Promega) was used to assess caspase activity. Cell apoptosis was quantified using a MACSQuant flow cytometer following double staining with the APC-Annexin V and 7-aminoactinomycin D (7-AAD) detection kit (BD Biosciences).

\section{Western blotting and quantitative reverse transcription PCR (RT-qPCR)}

To detect cellular protein levels, cell pellets were harvested and lysed by sonication in RIPA buffer (25mM Tris- $\mathrm{HCl} \mathrm{pH} 7.6,150 \mathrm{mM} \mathrm{NaCl}, 1 \% \mathrm{NP}-40$, $1 \%$ sodium deoxycholate, $0.1 \%$ SDS, plus $1 \mathrm{x}$ protease inhibitor cocktail). Lysate protein concentration was measured using a Pierce BCA Protein Assay kit (Life technologies). Approximately $40-60 \mu \mathrm{g}$ of protein for each sample was resolved on a $10 \%$ or $12 \%$ SDSPAGE gel and then transferred onto a nitrocellulose membrane for western blotting. The following primary antibodies were used for detection: glyceraldehyde3-phosphate dehydrogenase (GAPDH) (Fitzgerald, 10R-G109a), TfR1 (Life Technology, 13-6800), ferritin $\mathrm{H}$ monoclonal (hybridoma; Harlan Bioproducts for Science) and polyclonal [47], IRP1 (MediMabs, MM0074), IRP2 (Santa Cruz Biotechnology, sc-33682), Beta
Actin (Abcam, ab6276), p15 (Santa Cruz Biotechnology, SC612), p21 (Becton Dickinson, 556430), p27 (Cell Signaling Technology, \#3698)), p53 (Calbiochem Research Biochemicals, OP43). Goat anti-rabbit-HRP (Bio-Rad Laboratories, 170-6515) and goat anti-mouseHRP (Bio-Rad Laboratories, 170-6516) secondary antibodies were used.

RT-qPCR experiments were conducted essentially as described previously [13]. Briefly, RNA was extracted using the High Pure RNA Isolation Kit (Roche). cDNA was produced using the TaqMan ${ }^{\circledR}$ Reverse Transcription kit (Applied Biosystems). RT-qPCR was performed on a $V_{\text {ViiA }}{ }^{\text {TM }} 7$ Real-Time PCR System (Applied Biosystems) using a SYBR green-based PCR method. Primer sequences are listed in Supplementary Table 1. Experiments were performed 3 times with samples in triplicates. Representative results are shown. Comparative $\mathrm{Ct}$ method was used to calculate relative gene expression.

\section{Labile iron pool (LIP) assay}

The labile iron pool was measured using calcein AM ( $2 \mu \mathrm{M}, 15 \mathrm{~min}$ incubation) as a fluorescent probe for iron content. The concentration of calcein-bound iron was determined following addition of salicyladehydeisonicotinoyl-hydrazone $(10 \mu \mathrm{M})$ essentially as described [48].

\section{Transferrin binding assay}

To perform the transferrin binding assay, $\mathrm{LNCaP}$ cells were incubated with $50 \mu \mathrm{g} / \mathrm{ml}$ Alexa Fluor 647 labeled transferrin (Life Technologies). After a 20 minute incubation, cells were gently trypsinized and washed 3 times with 1x PBS to minimize non-specific binding of fluorescent transferrin. The fluorescent intensity of the harvested cells was determined by flow cytometer.

\section{Xenograft experiments}

Animal protocols were approved by the Institutional Animal Care and Use Committee (IACUC) at the University of Connecticut Health Center. 2 x $10^{6}$ PC3-Luc cells (infected with scrambled control or IRP2 shRNA lentiviruses) were suspended in $200 \mu \mathrm{l}$ of matrigel and injected subcutaneously into the rear flank of athymic nude mice (The Jackson Lab). Twelve mice were used in each group, however one mouse was excluded from the IRP2 knockdown group due to a failure to form a tumor. Tumor growth was monitored by digital calipers and bioluminescent luciferase imaging using an IVIS Spectrum (Perkin Elmer). Bioluminescent luciferase imaging was acquired and analyzed using Living Imaging 4.4 software (Perkin Elmer). Tumor volumes (V) were calculated by the formula $\mathrm{V}=\pi / 6 \times \mathrm{L} \times \mathrm{W}^{2}$ (L: length, $\mathrm{W}$ : width). 


\section{Statistical analyses}

In vitro experiments were performed at least three times. Experimental data are presented as mean \pm $\mathrm{SD}$, unless otherwise indicated. Statistical analysis was performed using GraphPad Prism (Graphpad Software). Student's t test (two-tailed) was used to evaluate the significance of observed differences between two groups. A p-value $<0.05$ was considered statistically significant (* $\mathrm{p}<.05, * * \mathrm{p}<.01, * * * \mathrm{p}<.001)$.

\section{Abbreviations}

7-AAD: 7-aminoactinomycin D; DFO: desferoxamine, an iron chelator; FPN: ferroportin; FTH: ferritin heavy chain; GAPDH: glyceraldehyde3-phosphate dehydrogenase; IACUC: Institutional Animal Care and Use Committee; IREs: iron responsive elements; IRPs: iron-responsive element-binding proteins; KD: knockdown; Luc: luciferase; p15: cyclin dependent kinase inhibitor 2B; p21: cyclin-dependent kinase inhibitor 1A; p27: cyclin dependent kinase inhibitor 1B; p53: tumor protein p53; PrEC: normal prostate epithelial cells; RT-qPCR: quantitative reverse transcription polymerase chain reaction; shRNA: short hairpin RNA; shCtr: shRNA control; TfR: transferrin receptor; UTR: untranslated region; WST-1: water soluble tetrazolium salt-1.

\section{Author contributions}

ZD performed the research. ZD, DHM, SVT, and FMT wrote and edited the manuscript. ZD, SVT, and FMT developed the concept and designed the experiments. The final manuscript was read and approved by all authors.

\section{ACKNOWLEDGMENTS}

We are grateful to Erica Lemler for her generous assistance with in vivo experiments and Dr. Evan Jellison for the kind help in flow cytometry analysis.

\section{CONFLICTS OF INTEREST}

We declare no conflicts of interest.

\section{FUNDING}

This work was supported in part by grants F30 DE026380 and T90 DE021989 from the NIDCR (D.H.M.), and NCI grants R01 CA188025 (S.V.T), and R01 CA171101 (F.M.T).

\section{REFERENCES}

1. Torti SV, Torti FM. Iron and cancer: more ore to be mined. Nat Rev Cancer. 2013; 13:342-355.

2. Winterbourn CC. Toxicity of iron and hydrogen peroxide: the Fenton reaction. Toxicol Lett. 1995; 82-83:969-974.

3. Lane DJ, Merlot AM, Huang ML, Bae DH, Jansson PJ, Sahni S, Kalinowski DS, Richardson DR. Cellular iron uptake, trafficking and metabolism: key molecules and mechanisms and their roles in disease. Biochim Biophys Acta. 2015; 1853:1130-1144.

4. Wang J, Pantopoulos K. Regulation of cellular iron metabolism. Biochem J. 2011; 434:365-381.

5. Thomson AM, Rogers JT, Leedman PJ. Iron-regulatory proteins, iron-responsive elements and ferritin mRNA translation. Int J Biochem Cell Biol. 1999; 31:1139-1152.

6. Leibold EA, Munro HN. Cytoplasmic protein binds in vitro to a highly conserved sequence in the $5^{\prime}$ untranslated region of ferritin heavy- and light-subunit mRNAs. Proc Natl Acad Sci U S A. 1988; 85:2171-2175.

7. Samaniego F, Chin J, Iwai K, Rouault TA, Klausner RD. Molecular characterization of a second iron-responsive element binding protein, iron regulatory protein 2 . Structure, function, and post-translational regulation. J Biol Chem. 1994; 269:30904-30910.

8. Miller KD, Siegel RL, Lin CC, Mariotto AB, Kramer JL, Rowland JH, Stein KD, Alteri R, Jemal A. Cancer treatment and survivorship statistics, 2016. CA Cancer J Clin. 2016; 66:271-289.

9. Pinnix ZK, Miller LD, Wang W, D'Agostino R Jr, Kute T, Willingham MC, Hatcher H, Tesfay L, Sui G, Di X, Torti $\mathrm{SV}$, Torti FM. Ferroportin and iron regulation in breast cancer progression and prognosis. Sci Transl Med. 2010; 2:43ra56.

10. Basuli D, Tesfay L, Deng Z, Paul B, Yamamoto Y, Ning G, Xian W, McKeon F, Lynch M, Crum CP, Hegde P, Brewer $\mathrm{M}$, Wang $\mathrm{X}$, et al. Iron addiction: a novel therapeutic target in ovarian cancer. Oncogene. 2017; 36:4089-4099.

11. Keer HN, Kozlowski JM, Tsai YC, Lee C, McEwan RN, Grayhack JT. Elevated transferrin receptor content in human prostate cancer cell lines assessed in vitro and in vivo. J Urol. 1990; 143:381-385.

12. Tesfay L, Clausen KA, Kim JW, Hegde P, Wang X, Miller LD, Deng Z, Blanchette N, Arvedson T, Miranti CK, Babitt JL, Lin HY, Peehl DM, et al. Hepcidin regulation in prostate and its disruption in prostate cancer. Cancer Res. 2015; 75:2254-2263.

13. Wang W, Deng Z, Hatcher H, Miller LD, Di X, Tesfay L, Sui G, D'Agostino RB Jr, Torti FM, Torti SV. IRP2 regulates breast tumor growth. Cancer Res. 2014; 74:497-507.

14. Muckenthaler MU, Galy B, Hentze MW. Systemic iron homeostasis and the iron-responsive element/ironregulatory protein (IRE/IRP) regulatory network. Annu Rev Nutr. 2008; 28:197-213. 
15. Meyron-Holtz EG, Ghosh MC, Iwai K, LaVaute $\mathrm{T}$, Brazzolotto X, Berger UV, Land W, Ollivierre-Wilson $\mathrm{H}$, Grinberg A, Love P, Rouault TA. Genetic ablations of iron regulatory proteins 1 and 2 reveal why iron regulatory protein 2 dominates iron homeostasis. EMBO J. 2004; 23:386-395.

16. Wang W, Di X, D'Agostino RB Jr, Torti SV, Torti FM. Excess capacity of the iron regulatory protein system. J Biol Chem. 2007; 282:24650-24659.

17. Sanchez M, Galy B, Schwanhaeusser B, Blake J, BahrIvacevic T, Benes V, Selbach M, Muckenthaler MU, Hentze MW. Iron regulatory protein-1 and -2: transcriptome-wide definition of binding mRNAs and shaping of the cellular proteome by iron regulatory proteins. Blood. 2011; 118:e168-e179.

18. Hileti D, Panayiotidis P, Hoffbrand AV. Iron chelators induce apoptosis in proliferating cells. Br J Haematol. 1995; 89:181-187.

19. Vermes I, Haanen C, Steffens-Nakken H, Reutelingsperger C. A novel assay for apoptosis. Flow cytometric detection of phosphatidylserine expression on early apoptotic cells using fluorescein labelled Annexin V. J Immunol Methods. 1995; 184:39-51.

20. Miller LD, Coffman LG, Chou JW, Black MA, Bergh J, D'Agostino R Jr, Torti SV, Torti FM. An iron regulatory gene signature predicts outcome in breast cancer. Cancer Res. 2011; 71:6728-6737.

21. Henderson BR, Menotti E, Kuhn LC. Iron regulatory proteins 1 and 2 bind distinct sets of RNA target sequences. J Biol Chem. 1996; 271:4900-4908.

22. Salahudeen AA, Thompson JW, Ruiz JC, Ma HW, Kinch LN, Li Q, Grishin NV, Bruick RK. An E3 ligase possessing an iron-responsive hemerythrin domain is a regulator of iron homeostasis. Science. 2009; 326:722-726.

23. Vashisht AA, Zumbrennen KB, Huang X, Powers DN, Durazo A, Sun D, Bhaskaran N, Persson A, Uhlen M, Sangfelt O, Spruck C, Leibold EA, Wohlschlegel JA. Control of iron homeostasis by an iron-regulated ubiquitin ligase. Science. 2009; 326:718-721.

24. Moroishi T, Nishiyama M, Takeda Y, Iwai K, Nakayama KI. The FBXL5-IRP2 axis is integral to control of iron metabolism in vivo. Cell Metab. 2011; 14:339-351.

25. Chang AH, Jeong J, Levine RL. Iron regulatory protein 2 turnover through a nonproteasomal pathway. J Biol Chem. $2011 ; 286: 23698-23707$

26. Fleming WH, Hamel A, MacDonald R, Ramsey E, Pettigrew NM, Johnston B, Dodd JG, Matusik RJ. Expression of the c-myc protooncogene in human prostatic carcinoma and benign prostatic hyperplasia. Cancer Res. 1986; 46:1535-1538.

27. Rebello RJ, Pearson RB, Hannan RD, Furic L. Therapeutic approaches targeting MYC-driven prostate cancer. Genes (Basel). 2017; 8.
28. Wu KJ, Polack A, Dalla-Favera R. Coordinated regulation of iron-controlling genes, H-ferritin and IRP2, by c-MYC. Science. 1999; 283:676-679.

29. King KL, Cidlowski JA. Cell cycle regulation and apoptosis. Annu Rev Physiol. 1998; 60:601-617.

30. Sobel RE, Sadar MD. Cell lines used in prostate cancer research: a compendium of old and new lines - part 1. J Urol. 2005; 173:342-359.

31. Tai S, Sun Y, Squires JM, Zhang H, Oh WK, Liang CZ, Huang J. PC3 is a cell line characteristic of prostatic small cell carcinoma. Prostate. 2011; 71:1668-1679.

32. Horoszewicz JS, Leong SS, Kawinski E, Karr JP, Rosenthal H, Chu TM, Mirand EA, Murphy GP. LNCaP model of human prostatic carcinoma. Cancer Res. 1983; 43:1809-1818.

33. Carroll AG, Voeller HJ, Sugars L, Gelmann EP. p53 oncogene mutations in three human prostate cancer cell lines. Prostate. 1993; 23:123-134.

34. Agarwal ML, Agarwal A, Taylor WR, Stark GR. p53 controls both the G2/M and the G1 cell cycle checkpoints and mediates reversible growth arrest in human fibroblasts. Proc Natl Acad Sci U S A. 1995; 92:8493-8497.

35. van Bokhoven A, Varella-Garcia M, Korch C, Johannes WU, Smith EE, Miller HL, Nordeen SK, Miller GJ, Lucia MS. Molecular characterization of human prostate carcinoma cell lines. Prostate. 2003; 57:205-225.

36. Kalinowski DS, Stefani C, Toyokuni S, Ganz T, Anderson GJ, Subramaniam NV, Trinder D, Olynyk JK, Chua A, Jansson PJ, Sahni S, Lane DJ, Merlot AM, et al. Redox cycling metals: pedaling their roles in metabolism and their use in the development of novel therapeutics. Biochim Biophys Acta. 2016; 1863:727-748.

37. Chitambar CR. The therapeutic potential of iron-targeting gallium compounds in human disease: from basic research to clinical application. Pharmacol Res. 2017; 115:56-64.

38. Le NT, Richardson DR. The role of iron in cell cycle progression and the proliferation of neoplastic cells. Biochim Biophys Acta. 2002; 1603:31-46.

39. Yu Y, Kovacevic Z, Richardson DR. Tuning cell cycle regulation with an iron key. Cell Cycle. 2007; 6:1982-1994.

40. Abeysinghe RD, Greene BT, Haynes R, Willingham MC, Turner J, Planalp RP, Brechbiel MW, Torti FM, Torti SV. p53-independent apoptosis mediated by tachpyridine, an anti-cancer iron chelator. Carcinogenesis. 2001; 22:1607-1614.

41. Greene BT, Thorburn J, Willingham MC, Thorburn A, Planalp RP, Brechbiel MW, Jennings-Gee J, Wilkinson J 4th, Torti FM, Torti SV. Activation of caspase pathways during iron chelator-mediated apoptosis. J Biol Chem. 2002; 277:25568-25575.

42. Simonart T, Boelaert JR, Mosselmans R, Andrei G, Noel JC, De Clercq E, Snoeck R. Antiproliferative and apoptotic 
effects of iron chelators on human cervical carcinoma cells. Gynecol Oncol. 2002; 85:95-102.

43. Turner J, Koumenis C, Kute TE, Planalp RP, Brechbiel MW, Beardsley D, Cody B, Brown KD, Torti FM, Torti SV. Tachpyridine, a metal chelator, induces G2 cell-cycle arrest, activates checkpoint kinases, and sensitizes cells to ionizing radiation. Blood. 2005; 106:3191-3199.

44. Daniels TR, Bernabeu E, Rodriguez JA, Patel S, Kozman M, Chiappetta DA, Holler E, Ljubimova JY, Helguera G, Penichet ML. The transferrin receptor and the targeted delivery of therapeutic agents against cancer. Biochim Biophys Acta. 2012; 1820:291-317.
45. Sui G, Shi Y. Gene silencing by a DNA vector-based RNAi technology. Methods Mol Biol. 2005; 309:205-218.

46. Deng Z, Wan M, Cao P, Rao A, Cramer SD, Sui G. Yin Yang 1 regulates the transcriptional activity of androgen receptor. Oncogene. 2009; 28:3746-3757.

47. Pietsch EC, Chan JY, Torti FM, Torti SV. Nrf2 mediates the induction of ferritin $\mathrm{H}$ in response to xenobiotics and cancer chemopreventive dithiolethiones. J Biol Chem. 2003; 278:2361-2369.

48. Epsztejn S, Kakhlon O, Glickstein H, Breuer W, Cabantchik I. Fluorescence analysis of the labile iron pool of mammalian cells. Anal Biochem. 1997; 248:31-40. 